\title{
On a Visibility Representation of Graphs
}

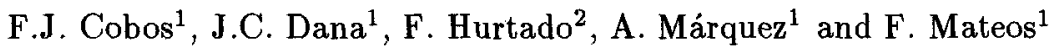 \\ 1 Universidad de Sevilla, Facultad de Informática y Estadística, Depto. de \\ Matemática Aplicada I, Sevilla, Spain \\ 2 Universidad Politécnica de Cataluña, Depto. de Matemática Aplicada II, \\ Barcelona, Spain
}

\begin{abstract}
We give a visibility representation of graphs which extends some very well-known representations considered extensively in the literature. Concretely, the vertices are represented by a collection of parallel hyper-rectangles in $\mathbf{R}^{n}$ and the visibility is orthogonal to those hyperrectangles. With this generalization, we can prove that each graph admits a visibility representation. But, it arises the problem of determining the minimum Euclidean space where such representation is possible. We consider this problem for concrete well-known families of graphs such as planar graphs, complete graphs and complete bipartite graphs.
\end{abstract}

\section{Introduction}

The problem of determining a visibility representation of a graph has been studied extensively in the literature due to the large number of applications (as in VLSI design, CASE tools, hidden-surface elimination problem, etc., [7, 8, 11, 13, 15]) and, also, by the combinatorial properties of those graphs.

In a visibility representation of a graph, the vertices map to objects in Euclidean space and the edges are determined by certain visibility relations.

Of course, both, the objects and the visibility used play an important role in characterizing the types of graphs that admit visibility representations. But, in any case, given a certain class of objects and a concrete visibility, there exist always graphs that are not representable, in this way, Tamassia \& Tollis [14] and Wismath [16] proved that a graph is a bar visibility graph (where the vertices represent horizontal line segments in the plane and two nodes are connected by an edge if their two horizontal rectangles can see each other vertically and non-degenerately) if and only if it admits a planar embedding with all cutpoints in the exterior face. And Bose et al. [3] proved that $K_{n}$ is not VR-representable for $n \geq 103$ (a graph is said to be VR-representable if each vertex of the graph maps to a closed rectangle in $\mathbf{R}^{3}$ such that the rectangles are disjoint, the planes determined by the rectangles are perpendicular to the $z$-axis, and the sides are parallel to the $x$ or $y$ axes. And, again, two nodes are connected by an edge if their two horizontal bars can see each other vertically and non-degenerately).

On the other hand, from a more theoretical point of view and since Kuratowski's Theorem [10], several measures of the planarity and/or dimension of a graph have been considered. But, few of these measures, notably, Boxicity, Grid 
intersection graphs $[1,9,2]$, are related with visibility representations in the line of the approachs mentioned above.

In this paper, we prove that the representations studied by Tamassia \& Tollis [14] and Wismath [16] in $\mathbf{R}^{2}$ and by Bose et al. [3] in $\mathbf{R}^{3}$ can be easily generalized to any dimension, and, if we consider for each graph the minimum $n$ where it is possible such a representation we obtain, in this way, a new measure of the complexity of the graph. Thus, we say that a graph is representable in $\mathbf{R}^{n}$ if it can be represented in such a way that each vertex maps to a hyperrectangle in $\mathbf{R}^{n}$ (where the hyper-rectangles that we consider are a cartesian product of $n-1$ closed intervals in $\mathbf{R}$ and a number in the last coordinate; i.e., $\left.\left[a_{1}, b_{1}\right] \times\left[a_{2}, b_{2}\right] \times \cdots \times\left[a_{n-1}, b_{n-1}\right] \times\left\{a_{n}\right\}\right)$ and two nodes are connected by an edge if there exists a closed cylinder in $\mathbf{R}^{n}$, orthogonal to the rectangles, of nonzero length and radius such that the ends of the cylinder are contained in each of the hyper-rectangles and it does not intersect any of the other hyper-rectangles. We say that a graph $G$ has representation index equal to $n$ (or $R I(G)=n$ for short) if $\mathbf{R}^{n}$ is the minimum where such a representation is possible.

This paper is organized in the following way, in Sect. 2 we prove that any graph has a finite $R I$, and we see that it is convenient to extend that index. Section 3 is devoted to the study of the $R I$ of planar graphs. And in Sect.4 and 5 we deal with the $R I$ of complete graphs and bipartite graphs respectively.

\section{Representation Index}

In this section we are going to prove that each graph is representable in some $\mathbf{R}^{n}$. First, we need the following lemma.

Lemma 1. Every graph representable in $\mathbf{R}^{n}$ is representable in $\mathbf{R}^{n+1}$.

Proof. It is easy to check that of the configuration

$$
R_{i}=\left[a_{1}^{i}, b_{1}^{i}\right] \times \cdots \times\left[a_{n-1}^{i}, b_{n-1}^{i}\right] \times\left\{a_{i}\right\} \quad i=1,2, \ldots p
$$

represent $G$, then the configuration

$$
R_{i}=\left[a_{1}^{i}, b_{1}^{i}\right] \times \cdots \times\left[a_{n-1}^{i}, b_{n-1}^{i}\right] \times[0,1] \times\left\{a_{i}\right\} \quad i=1,2, \ldots p
$$

also represents $G$.

Theorem 2. Given a graph $G$, there exists $n \in \mathbf{N}$ such that $G$ is representable in $\mathbf{R}^{n}$.

Proof. We will prove the theorem by induction on $k$ the number of vertices of $G$. Obviously the statement is true for small values of $k$. We now assume that $G$ has $k+1$ vertices and that the statement is true for graphs with $k$ vertices. Given a vertex $p$ of $G$, we split a representation $\mathcal{R}$ of $G-p$ into two subset

$$
\mathcal{R}=\left\{R_{t_{1}}, R_{t_{2}}, \ldots, R_{t_{n_{p}}}\right\} \cup\left\{Q_{u_{1}}, Q_{u_{2}}, \ldots, Q_{u_{\left(k-n_{p}\right)}}\right\}
$$




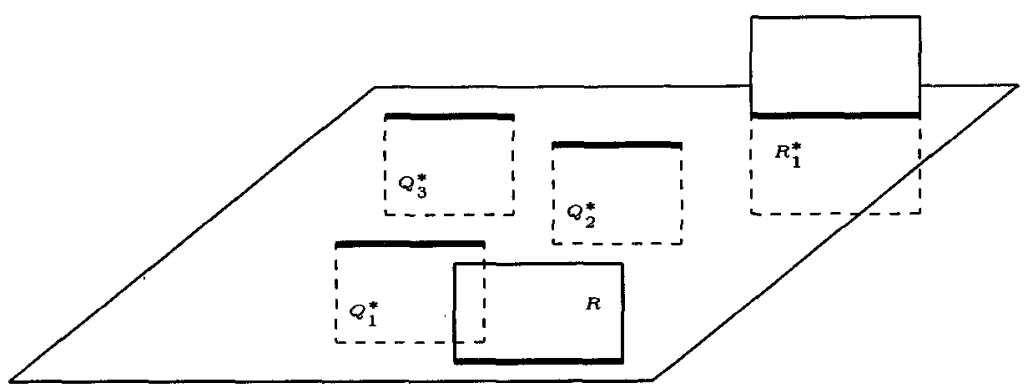

Fig. 1. $G$ is representable in $\mathbf{R}^{n}$.

In such a way that the hyper-rectangles $R_{t_{i}}=I_{t_{i} 1} \times \cdots \times I_{t_{i} n-1} \times\left\{t_{i}\right\}(1 \leq i \leq$ $n_{p}$ ) correspond with the vertices $p_{t_{i}}$ adjacent to $p$ in $G$. Now, it is easy to see that the configuration $\mathcal{R}^{*}=\left\{R_{t_{1}}^{*}, R_{t_{2}}^{*}, \ldots, R_{t_{n_{p}}}^{*}\right\} \cup\left\{Q_{u_{1}}^{*}, Q_{u_{2}}^{*}, \ldots, Q_{u_{\left(k-n_{p}\right)}}^{*}\right\} \cup\{R\}$ represents $G$ (being $p$ represented by $R$ ), where

$$
\begin{aligned}
& Q_{u_{j}}^{*}=\mathcal{I}_{u_{j} 1} \times \cdots \times \mathcal{I}_{u_{j} n-1} \overbrace{\times[-1,0] \times \cdots \times[-1,0]}^{n_{p}} \times\left\{u_{j}\right\} \\
& R_{t_{1}}^{*}=I_{t_{1} 1} \times \cdots \times I_{t_{1} n-1} \overbrace{\times[-1,1] \times \cdots \times[-1,0] \times \cdots \times[-1,0]}^{n_{p}} \times\left\{t_{1}\right\} \\
& \vdots \\
& R_{t_{s}}^{*}=I_{t_{s} 1} \times \cdots \times I_{t_{s} n-1} \overbrace{\times[-1,0] \times \cdots \times[-1,1] \times \cdots \times[-1,0]}^{n_{p}} \times\left\{t_{s}\right\} \\
& \quad \vdots \\
& R_{t_{n_{p}}}^{*}=I_{t_{n_{p}} 1} \times \cdots \times I_{t_{n_{p}}} n-1 \overbrace{\times[-1,0] \times \cdots \times[-1,0] \times \cdots \times[-1,1]}^{n_{p}} \times\left\{t_{n_{p}}\right\} \\
& \text { and } R=I_{1} \times \cdots \times I_{n-1} \overbrace{\times[0,1] \times \cdots \times[0,1] \times \cdots \times[0,1]}^{n_{p}} \times\left\{t_{k+1}\right\} \text { with } I_{i}=
\end{aligned}
$$
$\left[\min _{j}\left\{x: x \in I_{j i}\right\}, \max _{j}\left\{x: x \in I_{j i}\right\}\right]$.

Observe, that in Lemma 1 the last interval in all hyper-rectangles in the configuration is always $[0,1]$, that means that we are not using the whole $\mathbf{R}^{n}$ for our representation but only a half $\left(x_{n-1} \geq 0\right)$ and that all hyper-rectangles are lying on the hyper-plane of equation $x_{n-1}=0$. Thus, we can give a finer definition of $R I$ saying that a graph $G$ of (old) representation index equal to $n$ has actually $R I(G)=(n-1)+1 / 2$ if it admits a reprepresentation in $\mathbf{R}^{n}$ such that any hyper-rectangle is of the form $\left[a_{1}, b_{1}\right] \times\left[a_{2}, b_{2}\right] \times \cdots \times\left[0, b_{n-1}\right] \times\left\{a_{n}\right\}$.

This new definition will allow us to get a better view of the problem, and we will get our main results using it. Moreover, there are some other additional reasons to consider this more general concept of representation index. For 

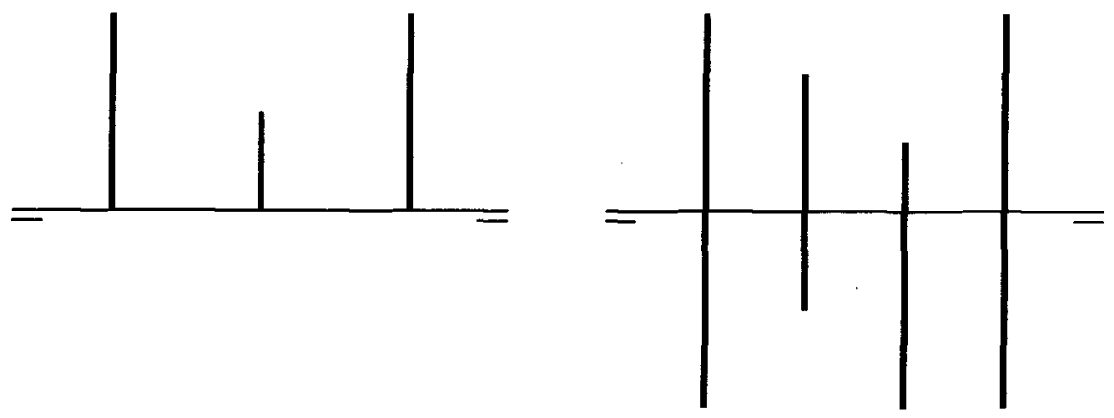

Fig. 2. $R I\left(K_{3}\right)=1+1 / 2$ and $R I\left(K_{4}\right)=2$.

instance, observe that if a graph has index $1+1 / 2$, then we can associate it an $n$-tuple of integer numbers in such a way that each vertex maps to one of the number of the $n$-tuple and two vertices are joined by an edge if all numbers between them in the $n$-tuple are smaller. Note that graphs with index $1+1 / 2$ are not the same of those of index $2\left(K_{4}\right.$ has index 2 and $K_{3}$ has index $\left.1+1 / 2\right)$.

\section{Planar Graphs}

Bose et al. [3] proved that if $G$ is planar then $R I(G) \leq 3$. On the other hand, Tamassia \& Tollis [14] and Wismath [16] gave the following theorem characterizing those graphs with representation index smaller or equal to 2 .

Theorem 3. [14, 16] A graph $G$ has representation index smaller or equal to 2 if and only if there is a planar embedding of $G$ with all cutpoints on the exterior face.

We complete now this theorem, characterizing those graphs with representation index $1+1 / 2$. For that characterization we say that a graph $G$ is outerhamiltonian if it has a path (possibly open) containing all vertices of $G$ such that there exists a planar embedding of $G$ with all edges of that path on the exterior face. Observe that Mitchell's algorithm to determine if a graph is outerplanar [12] with some modifications allows to get a linear-time algorithm to determine if a given graph is outerhamiltonian.

Theorem 4. A graph has representation index $1+1 / 2$ if and only if it is outerhamiltonian.

Proof. It is obvious that if $R I(G)=1+1 / 2$ then $G$ is outerhamiltonian.

For the sufficiency there are two cases to consider.

In the first case, we suppose that $G$ is 2 -connected. In this case, $G$ is a polygon of vertices $\left\{v_{1}, v_{2}, \ldots, v_{n}\right\}$ (where that ordering if one of the two possible orderings of the vertices of the polygon) with some of its non-intersecting diagonals. 


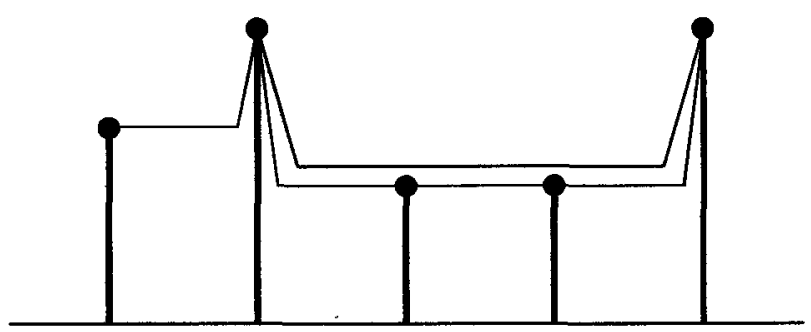

Fig. 3. If $R I(G)=1+1 / 2$ then $G$ is outerhamiltonian.

We map the vertex $v_{i}$ to the bar $\left[0, d_{i}\right] \times\left\{a_{i}\right\}$, where $d_{i}-1$ for $2 \leq i \leq n-1$ is the total number of diagonals minus the number of diagonals $\left\{v_{l}, v_{k}\right\}$ with $l<i<k$, and $d_{1}=d_{n}$ is the total number of diagonals plus 2 .

In the second case, if $G$ is not 2-connected, in each block there exist, at most, two cutpoints, place them the first and the last in that block, and we sort the blocks, obtaining, in that way, an ordering of all vertices of the graph. Now, we represent each block as in the first case, but giving the same length (the biggest one) to all bars representing cut-points.
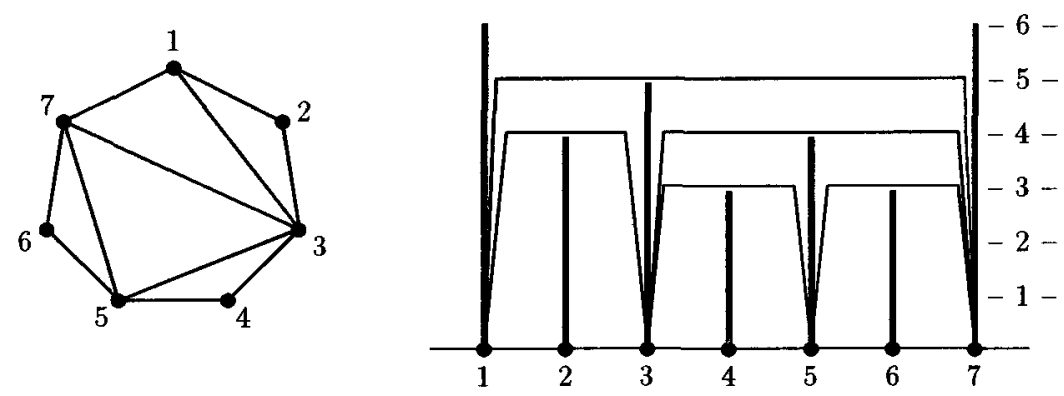

Fig. 4. Construction of a bar-representation in $\mathbf{R}^{1+1 / 2}$

Finally Bose et al.[3] proved that if $G$ is a planar graph then $R I(G) \leq 3$. Thus, it remains to determine which planar graphs have representation index $2+1 / 2$, this question is still open.

\section{Complete Graphs}

As it was said before $R I\left(K_{3}\right)=1+1 / 2, R I\left(K_{4}\right)=2$ and Bose et al. [3] proved that if $n \leq 20$ then $R I\left(K_{n}\right) \leq 3$. In this section, we are going to prove that $R I\left(K_{10}\right)=2+1 / 2$ and that $R I\left(K_{2 n}\right) \leq R I\left(K_{n}\right)+1 / 2$. Observe that this 
implies Bose et al. [3] result as a particular case. Moreover, we give lowerbounds to the representation index of complete graphs.

Lemma 5. $R I\left(K_{10}\right)=2+1 / 2$.

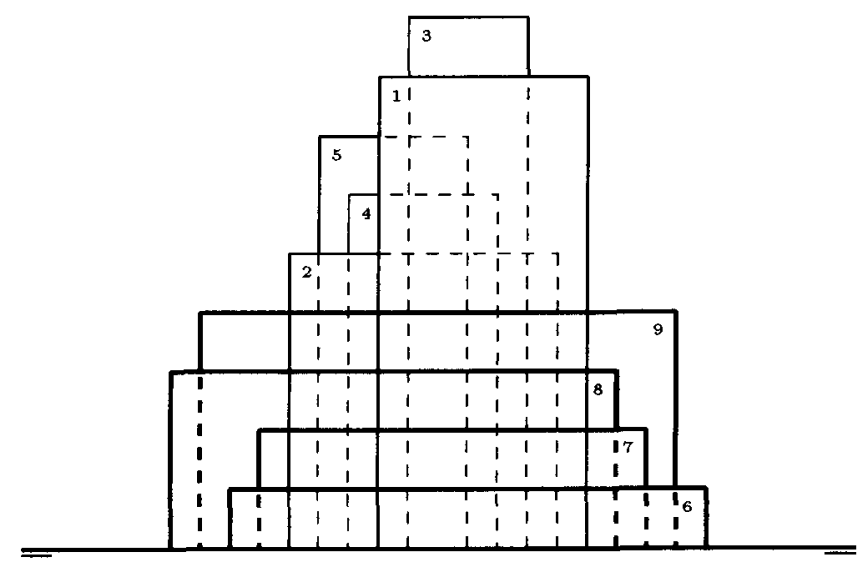

Fig. 5. $K_{10}$ in $\mathbf{R}^{2+1 / 2}$

Proof. We can give the following configuration (Fig. 5) of 9 rectangles, such that all them are seen from the front.

Theorem 6. $R I\left(K_{2 n}\right) \leq R I\left(K_{n}\right)+1 / 2$.

Proof. (Outline) We consider two cases. In the first one, we assume that $R I\left(K_{n}\right)$ is not an integer number. If we have a collection of $n$ hyper-rectangles in a half of $\mathbf{R}^{m}$ representing $K_{n}$ and such that the last interval of each one is $\left[0, a_{i}\right]$, then we consider a new configuration sustituing those intervals by $\left[-i, a_{i}\right]$, we get a representation of $K_{2 n}$ enfrenting to that configuration a new copy of that configuration but flipper over and rotated $90^{\circ}$ (see Fig.6).

In the second case, we suppose that $R I\left(K_{n}\right)$ is an integer number. If we have a collection of $n$ hyper-rectangles in $\mathbf{R}^{m}$ representing $K_{n}$, then it must exist a hyper-plane ortogonal to all hyper-rectangles and intersecting all them. Thus, we can suppose that $x_{m-1}=0$ is such a hyperplane. That means that the $i$-th hyper-rectangle ends with the product $\left[a_{i}, b_{i}\right] \times\left\{a_{i}\right\}$ with $a_{i}<0<b_{i}$, then we sustitute those products by $\left[-i, b_{i}\right] \times\left[0,-a_{i}\right] \times\left\{a_{i}\right\}$. In this way, we get $n$ hyperrectangles in a half of $\mathbf{R}^{m+1}$ representing $K_{n}$ such that the intervals before the last intervals in the product defining those hyper-rectangle constitute a sort of staircase, we can face in front of them the same configuration, but now changing $\left[a_{i}, b_{i}\right] \times\left\{a_{i}\right\}$ by $\left[a_{i}, b_{i}\right] \times[0, i] \times\{-i\}$ in order to get a representation of $K_{2 n}$ in a half of $\mathbf{R}^{m+1}$ (see Fig.7). 

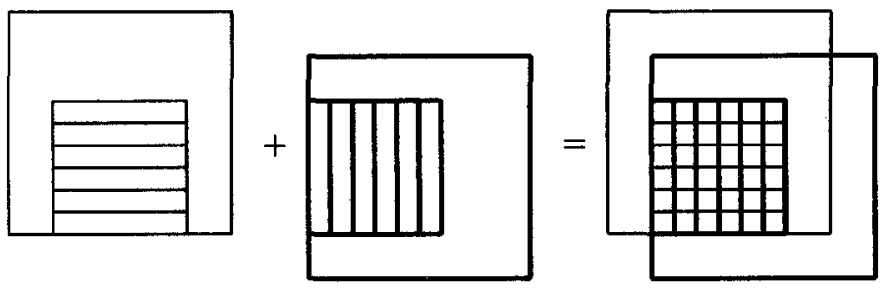

Fig. 6. The first case of Theorem 6 .

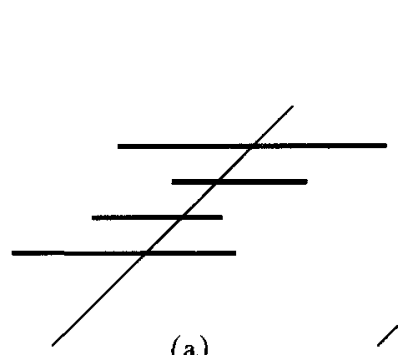

(a)

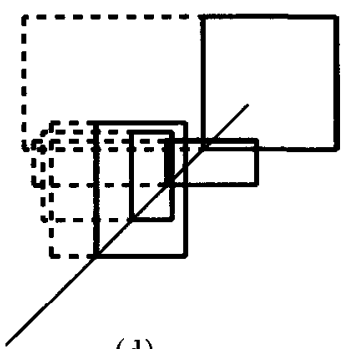

(d)

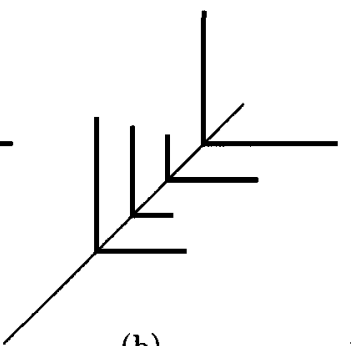

(b)

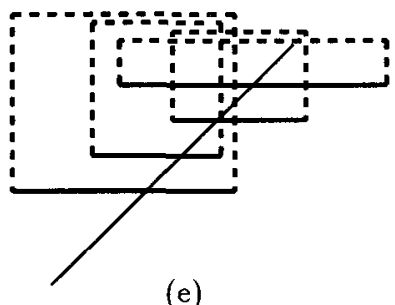

(e)

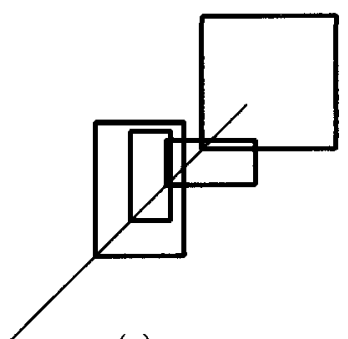

(c)

Fig. 7. The second case of Theorem 6 .

As an inmediate consequence of Lemma 5 and Theorem 6 we get.

Corollary 7. For all $n>2$ if $m \leq 5 \cdot 4^{n-2}$ then $R I\left(K_{m}\right) \leq n$.

Corollary 7 provides an upperbound to the representation index of the complete graphs. Now, we are going to try to get a lowerbound. As Fekete, Houle and Whitesides do in their paper [6], we will use the following lemma.

Lemma 8. [Attributed by F.R.K. Chung [4] to V. Chvátal and J.M. Steele, among others.] For all $m>1$, in every sequence of $\left(\begin{array}{c}m \\ 2\end{array}\right)+1$ distinct integers, there exists at last one strongly unimaximal subsequence (with only one local maximum) of length $m$ ). On the other hand, there exists a sequence of $\left(\begin{array}{c}m \\ 2\end{array}\right)$ distinct integers that has no strongly unimaximal subsequence of length $m$. 
We will use the following easy-to-prove lemma.

Lemma 9. If the hyper-rectangles $R_{i}=\left[a_{1}^{i}, b_{1}^{i}\right] \times\left[a_{2}^{i}, b_{2}^{i}\right] \times \cdots \times\left[a_{t-1}^{i}, b_{t-1}^{i}\right] \times\left\{a_{i}\right\}$ with $1 \leq i \leq n\left(b_{t-1}^{i} \geq 0\right)$ represent $K_{n}$, and $\left\{\left(a_{t-1}^{i}\right) \quad 1 \leq i \leq n\right\}$ is a strongly unimaximal sequence, then the hyper-rectangles $R_{i}^{\prime}=\left[a_{1}^{i}, b_{1}^{i}\right] \times\left[a_{2}^{i}, b_{2}^{i}\right] \times \cdots \times$ $\left[0, b_{t-1}^{i}\right] \times\left\{a_{i}\right\}$ represent also $K_{n}$.

Bose el al. prove in their paper [3] that $R I\left(K_{103}\right) \geq 3$ and Fekete, Houle \& Whitesides prove in [6] by using of Lemma 8 that $R I\left(K_{56}\right) \geq 3$. It is possible to get this same lowerbound as a consequence of Lemma 9 and Lemma 8, in fact, a more general result can be achieved.

Theorem 10. $R I\left(K_{\left(\begin{array}{c}m \\ 2\end{array}\right)+1}\right)>R I\left(K_{m}\right)$.

Proof. (Outline) If the hyper-rectangles $R_{i}=\left[a_{1}^{i}, b_{1}^{i}\right] \times\left[a_{2}^{i}, b_{2}^{i}\right] \times \cdots \times\left[a_{t-1}^{i}, b_{t-1}^{i}\right] \times$ $\left\{a_{i}\right\}\left(b_{t-1}^{i} \geq 0\right)$ with $1 \leq i \leq\left(\begin{array}{c}m \\ 2\end{array}\right)+1$ represent $K_{\left(\begin{array}{c}m \\ 2\end{array}\right)+1}$, by Lemma 8 , there exists a strongly unimaximal subsequence of $\left(a_{t-1}^{i}\right)$ of length $m$. Then Lemma 9 assures that $K_{n}$ has representation index strictly lower than $K_{\left(\begin{array}{c}m \\ 2\end{array}\right)+1}$.

\section{Complete Bipartite Graphs}

In this section we characterize the representation index of all complete bipartite graphs. Firstly, it is easy to observe that $R I\left(K_{n, m}\right) \leq 3$. But from the results in Sect. 3, we get that $R I\left(K_{1,2}\right)=R I\left(K_{2,2}\right)=1+1 / 2$, and that $R I\left(K_{2, n}\right)=$ $R I\left(K_{2, n}\right)=2$ for all $n>2$.

Now, we are going to prove that $R I\left(K_{3, n}\right)=2+1 / 2$, for $n \geq 3$, and that $R I\left(K_{n, m}\right)=3$ when $n, m \geq 4$.

Lemma 11. $R I\left(K_{3, n}\right)=2+1 / 2$, for $n \geq 3$.

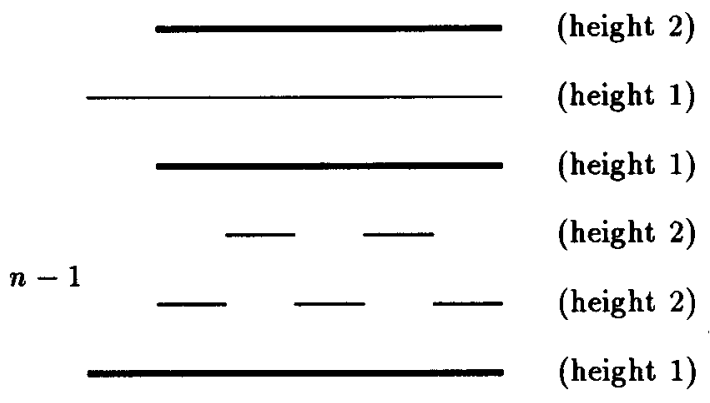

Fig. 8. $K_{3, n}$ in $\mathbf{R}^{2+1 / 2}$ 
Proof. As $K_{3, n}$ is not planar, we get that $R I\left(K_{3, n}\right) \geq 2+1 / 2$, and we can give the configuration of Fig. 8 to prove that $R I\left(K_{3, n}\right)=2+1 / 2$.

And studying exhaustly all cases it is possible to prove the following lemma.

Lemma 12. $R I\left(K_{4,4}\right)=3$.

Finally, it is trivial to check that $R I\left(K_{m, n}\right)=3$ for all $m, n \in \mathrm{N}$ such that $4 \leq m \leq n$.

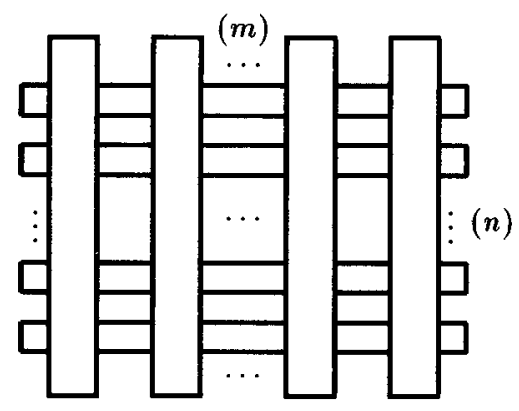

Fig. 9. $K_{n, m}$ in $\mathbf{R}^{3}$

\section{Conclusions}

We can sumarize our results in the following table.

\begin{tabular}{|c||c||c|}
\hline Type of graph $G$ & $R I(G)$ & Reference \\
\hline \hline Outerhamiltonian & $1+1 / 2$ & Theorem 4 \\
\hline Cutpoint-outerplanar & $\leq 2$ & Theorem 3 [14] \\
\hline Planar & $\leq 3$ & Bose et al. [3] \\
\hline \hline$K_{n} n \leq 3$ & $1+1 / 2$ & \\
\hline$K_{4}$ & 2 & \\
\hline$K_{10}$ & $2+1 / 2$ & Lemma 5 \\
\hline$K_{11}$ & 3 & Theorem 10 \\
\hline$K_{n} \quad n \geq 56$ & $>3$ & Fekete et al. [6] \\
\hline$K_{5 \cdot 4^{n-2}} n>2$ & $\leq n$ & Corollary 7 \\
\hline$K_{n} n \geq\left(\begin{array}{c}m \\
2\end{array}\right)+1$ & $>R I\left(K_{m}\right)$ & Theorem 10 \\
\hline \multicolumn{2}{|c}{} \\
\hline
\end{tabular}




\begin{tabular}{|c||c||c|}
\hline$K_{1,2}$ or $K_{2,2}$ & $1+1 / 2$ & \\
\hline$K_{1, n}$ or $K_{2, n} \quad n \geq 3$ & 2 & \\
\hline$K_{3, n} \quad n \geq 3$ & $2+1 / 2$ & Lemma 11 \\
\hline$K_{4,4}$ & 3 & Lemma 12 \\
\hline$K_{m, n} \quad 4 \leq m \leq n$ & 3 & Bose et al. [3] \\
\hline
\end{tabular}

\section{References}

1. H. Alt, M. Godau and S. Whitesides. Universal 3-Dimensional Visibility Representations for Graphs. Abstract presented at Graph Drawing'95, Passau (Germany). 1995.

2. S. Bellantoni, I. Ben-Arroyo Hartman, T. Przytycka and S. Whitesides. Grid intersection graphs and boxicity. North-Holland. Discrete Mathematics, 114:41-49, 1993.

3. P. Bose, H. Everett, S. Fekete, A. Lubiw, H. Meijer, K. Romanik, T. Shermer and S. Whitesides. On a Visibility Representation for Graphs in Three Dimensions. In David Avis and Prosenjit Bose, editors, Snapshots in Computational and Discrete Geometry, volume III, McGill University, July 1994. Technical Report SOCS-94.50.

4. F.R.K. Chung. On unimodal subsequences. J. Combinatorial Theory, Series A, v. $29: 267-279,1980$.

5. P. Erdös and A. Szekeres. A combinatorial problem in geometry. Compositio Mathematica, v. 2:463-470, 1935.

6. S.P. Fekete, M.E. Houle and S. Whitesides. New Results on a Visibility Representation of Graphs in 3D. Abstract presented at Graph Drawing'95, Passau (Germany). 1995.

7. M. Garey, D. Jhonson and H. So. An application of graph coloring to printed circuit testing. IEEE Trans. Circuits and Systems., CAS-23:591-598, 1976.

8. M.Y. Hsueh and D.O. Pederson. Computer-aided layout of LSI circuit bulding bloks. Proc. IEEE Int. Symp. on Circuits and Systems., pages 474-477, 1979.

9. J. Kratochvil and T. Przytycka. Grid intersection graphs and boxicity on surfaces. Abstract presented at Graph Drawing'95, Passau (Germany). 1995.

10. K. Kuratowski. Sur le problème des courbes gauches en topologie. Fund. Math., 15:271-283, 1930.

11. E. Lodi and L. Pagli. A VLSI algorithm for a visibility problem. In P. Bertolazzi and F. Luccio, editors, VLSI: Algorithms and Architectures, pages 125-134. NortHolland, Amsterdam, 1985.

12. S.L. Mitchell. Linear algorithms to recognize outerplanar and maximal outerplanar graphs. Information Processing Letters, 9(5):229-232, 1979.

13. M. Schlag, F. Luccio, P. Maestrini, D.T. Lee and C.K. Wong. A visibility problem in VLSI layout compaction. In F. P. Preparata, editor, Advances in Computing Research, volume 2, pages 259-282. JAI Press Inc, Greenwich. CT., 1985.

14. R. Tamassia and I.G. Tollis. A unified approach to visibility representations of planar graphs. Discrete and Computational Geometry, 1:321-341, 1986.

15. S. Wimer, I. Koren and I. Cederbaum. Floorplans, planar graphs and layouts. IEEE Trans. Circuits ans Systems, 35:267-278, 1988.

16. S.K. Wismath. Characterizing bar line-of-sight graphs. Proc. ACM Symp. on Computational Geometry. Baltimore. MD. 1985. 